\title{
Review of development and characterization of sugar palm fiber reinforced polymer composites
}

\begin{abstract}
Sugar palm fiber (SPF) has been gaining interest in the area of natural fiber composites owing to its good mechanical properties, high durability, good resistance to seawater and biodegradability. SPF is among the natural fibers that could be a potential material to be used as reinforcement in polymer composites. Incorporation of SPF as reinforcement in polymer composites has resulted in improvement in the physical, mechanical, and thermal properties of the composites. Another interesting point is the low density of SPF, which helps to reduce the weight of the composites, as well as increase the biodegradability. Much research has been carried out to determine the suitability of SPF as reinforcement in polymer composites. SPF-reinforced composites are suitable for a wide range of applications such as automotive anti-roll bars, rescue boats and drain covers. Chapter 2 provides an overview of the characteristics and development of various products derived from SPF-reinforced composites and the future direction of SPF and SPF-reinforced composites.
\end{abstract}

